\title{
Reliability Effects of X-Ray Lithography Exposures on Submicron-Channel MOSFETs*
}

\author{
Aivars J. Lelis and Timothy R. Oldham \\ U.S. Army Research Laboratory \\ 2800 Powder Mill Road \\ Adelphi, MD 20783-1197
}

\section{Abstract}

Submicron-channel-length $n$ - and $p$-channel MOSFETs subjected to channel hot-carrier stressing were investigated, and the reliability of devices with and without exposure to simulated $\mathrm{x}$-ray lithography processing steps was compared. No significant differences were observed between the sample groups.

\section{INTRODUCTION}

The ionizing radiation used in $\mathrm{x}$-ray lithography can be quite detrimental to semiconductor-based electronics, resulting in charge trapping at and near the insulating oxide interface of MOS devices [1-3], especially at the doses required for a typical $\mathrm{x}$-ray lithography mask level (50 to $100 \mathrm{Mrads}\left(\mathrm{SiO}_{2}\right)$ ). However, there are two mitigating reasons why the problem is not as great as some fear. First, the $\mathrm{x}$-ray exposures occur without an applied bias, if indeed the devices have electrodes yet. Second, the $\mathrm{x}$-ray exposures are typically followed with high-temperature annealing steps, which can repair most or all of the damage introduced by the radiation, such as oxide trapped holes and interface traps. However, the concern has been that some traps may become charged during operation (for example, from channel hot-carrier injection). Even though the damage appears to be annealed, some authors speculate that there might still be some impact on long-term reliability, e.g., from neutral electron traps (NETs) [4].

We discussed these concerns previously [5] when we compared the effects of channel hot carriers on both $n$-and $p$-channel devices with 1.0- $\mu \mathrm{m}$ channel lengths and found no significant operating shifts for either control devices or those simulating $\mathrm{x}$-ray lithography processing [5]. The goal of this work is to compare the reliability of devices with submicron channel lengths.

\section{Procedure}

Both $n$ - and $p$-channel metal-oxide semiconductor fieldeffect transistors (MOSFETs) have been examined that were processed by a major U.S. manufacturer using bulk Si radiationhardened technology with poly-Si gates. All the devices tested had channel widths of $10.0 \mu \mathrm{m}$ and varying channel lengths, from 1.0 to $0.5 \mu \mathrm{m}$. The gate oxides were $12.5 \mathrm{~nm}$ thick.

The devices were split into two sets of samples: the control set was not irradiated, and we irradiated the other set to a total dose of $50 \mathrm{Mrads}\left(\mathrm{SiO}_{2}\right)$ using $10-\mathrm{keV}$ x-rays with no bias ap-

*This work was partially funded by the (Defense) Advanced Research Projects Agency (ARPA). plied in order to simulate a typical $\mathrm{x}$-ray lithography mask level This exposure was followed by a 30-min anneal in forming gas $\left(10 \% \mathrm{H}_{2}, 90 \% \mathrm{~N}_{2}\right)$ at about $400^{\circ} \mathrm{C}$ to simulate a typical postmetal anneal (PMA).

These two sets of samples were then biased in various configurations that simulated both realistic and unrealistic potential channel hot-carrier conditions. The primary test condition was a DC channel hot-carrier stress, where the gate was biased at $1.6 \mathrm{~V}$ and the drain at $5.0 \mathrm{~V}$, with the source and substrate grounded. On average, we monitored the devices twice per decade of time using an HP4145B parametric analyzer to record drain current (with $0.15 \mathrm{~V}$ applied to the drain) versus applied gate voltage $\left(I_{D}-V_{G}\right)$ characteristics. We subsequently applied the standard subthreshold charge separation technique [6] to resolve $\Delta V_{T H}$ into oxide trapped charge and interface trapped charge components. The post-irradiation, pre-anneal $\Delta V_{T H}$ was on average about $0.5 \mathrm{~V}$. The post-anneal $\Delta V_{T H}$ decreased $95 \%$ on average, to about $30 \mathrm{mV}$.

\section{RESULTS AND Discussion}

\section{N-Channel Devices}

Figure 1 compares the threshold voltage shifts of both control devices and irradiated/annealed devices of $1.0-\mu \mathrm{m}$ channel length that were subjected to channel hot-carrier stress for over $10^{6} \mathrm{~s}$. (In all the figures, the solid symbol curves represent the responses of irradiated devices and the open symbol curves those of control devices.) No difference whatsoever is observed between the responses for the first $10^{4} \mathrm{~s}$, after which time both devices begin exhibiting a positive threshold voltage shift, $\Delta V_{T H}$,

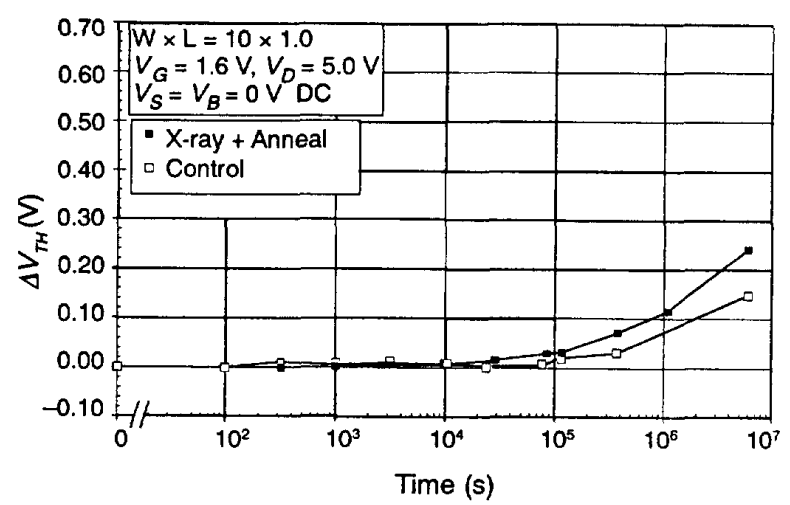

Figure 1. A comparison of threshold voltage shifts, $\Delta V_{T H}$, for both irradiated and annealed $1.0-\mu \mathrm{m} n$-channel devices and controls, during a $10^{6}$-s hot-carrier stress. 
with the device that was exposed to simulated x-ray lithography showing the slightly greater shift. This is a fairly typical result, in that both the irradiated/annealed and the control devices experience a positive $\Delta V_{T H}$ at late times, with the irradiated device having a slightly larger shift. Figure 2 shows the results of comparing the channel hot-carrier stress between two $0.5-\mu \mathrm{m}$ channel-length devices. These devices behave very similarly to the 1.0- $\mu \mathrm{m}$ devices of Figure 1. In fact, their responses would look almost identical if those in Figure 1 were translated about two decades earlier in time. Also, the irradiated devices begin shifting slightly earlier, but once the control devices begin shifting, they exhibit roughly the same response.

Figure 3 simply replots the results from Figures 1 and 2 along with the responses of both irradiated/annealed and control devices with channel lengths of $0.8,0.7$, and $0.6 \mu \mathrm{m}$, all on the same graph. The results from Figures 1 and 2 serve as bookends for the results of the other devices.

Two important facts are borne out: (1) shorter channel length devices are affected more (as expected) and, even more important, (2) similar increases in $\Delta V_{T H}$ are observed for both control devices and those irradiated and annealed to simulate the effects of x-ray lithography. The controls do shift less, but not significantly so. Primarily, we observe that failure is a consequence of device geometry and not of potential processing differences ( $x$-ray or optical lithography).

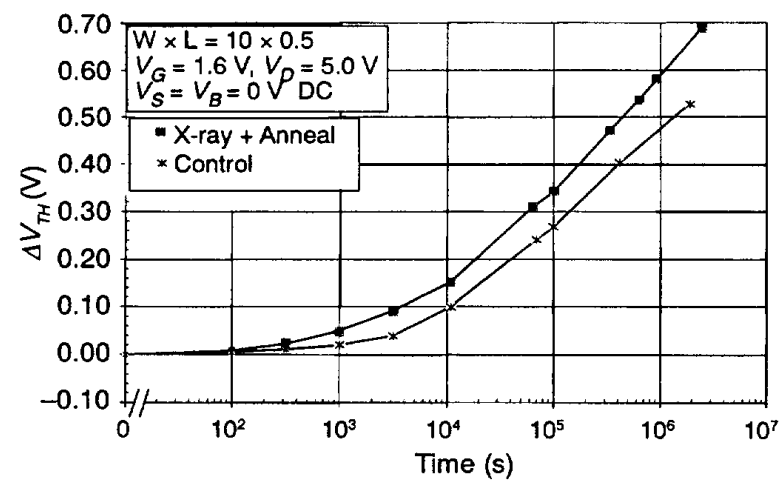

Figure 2. A comparison of threshold voltage shifts, $\Delta V_{T H}$, for both irradiated and annealed $0.5-\mu \mathrm{m} n$-channel devices and controls, during a $10^{6}$-s hot-carrier stress.

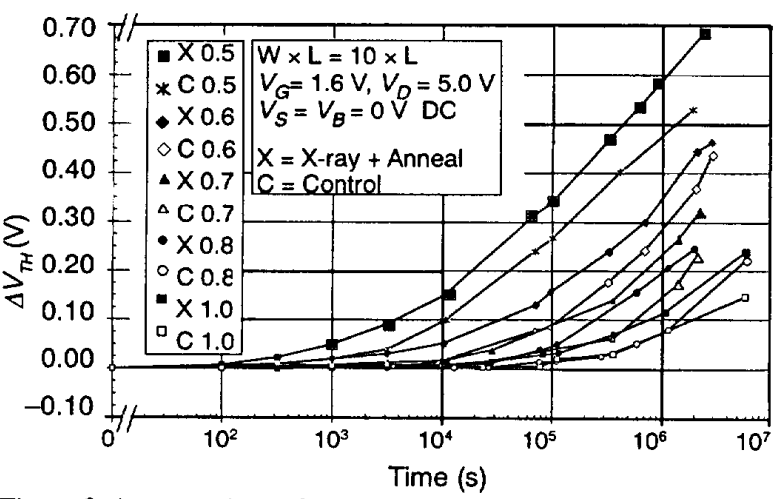

Figure 3. A comparison of threshold voltage shifts, $\Delta V_{T H}$, for both irradiated and annealed $n$-channel devices and controls, as a function of channel length, during a $10^{6}$-s hot-carrier stress.
The longer channel length devices shift less than the shorter channel length devices, because of the larger lateral electric field, $E_{m}$, in the devices with the shorter channel length. This electric field is localized near the drain end of the channel. When the device is biased "on," so that a current flows, these charge carriers are subsequently accelerated or "heated" by the localized field. They undergo impact ionization, creating electron-hole pairs. The holes are generally collected by the substrate, leading to several problems, but also providing a means for quantifying which bias conditions produce the greatest hot-carrier damage. Thus $n$-channel devices are customarily biased in what is called the maximum substrate current condition [7]. This is generally a little above threshold [8] because the channel must be turned fully on to allow the maximum number of charge carriers to flow. (In our case, we have stressed the devices shown in Figures 1 to 5 with a gate bias of $1.6 \mathrm{~V}$.) Countering this is that the maximum electric field, $E_{m}$, diminishes with gate bias (since the drain saturation voltage increases with gate bias), and the maximum electric field is proportional to the difference of the drain bias and the drain saturation bias $\left(E_{m} \propto V_{D}-V_{D} s a t\right)$ [9].

The more energetic charge carriers can cross the oxide energy barrier and either be collected by the gate electrode or become trapped in the oxide. They also can activate previously passivated interface traps, which is generally the main long-term defect mechanism in $n$-channel devices. These results are clearly

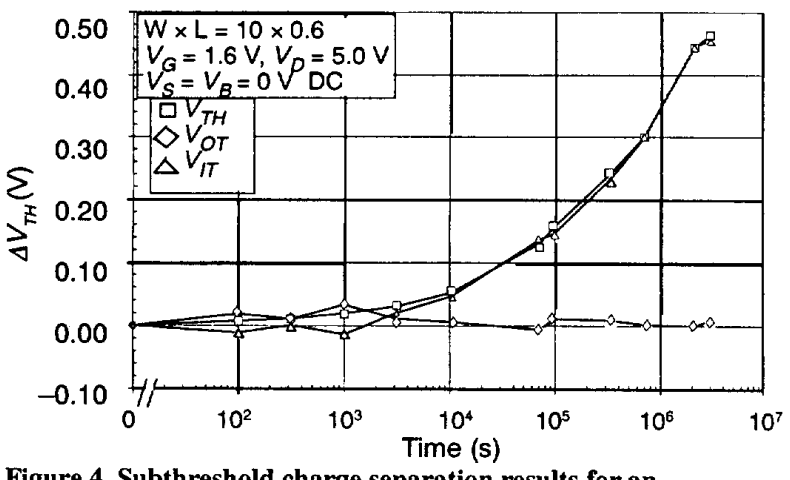

Figure 4. Subthreshold charge separation results for an irradiated and annealed $0.6-\mu \mathrm{m} n$-channel device subject to a channel hot-carrier stress for $10^{6} \mathrm{~s}$

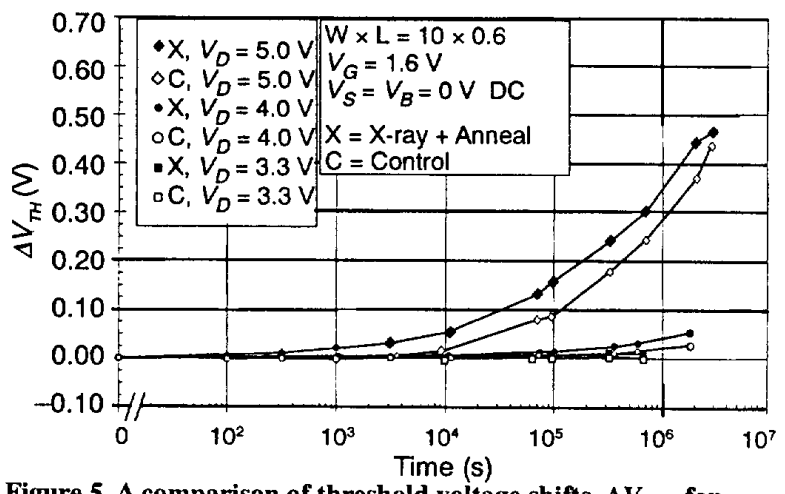

Figure 5. A comparison of threshold voltage shifts, $\Delta V_{T H}$, for both irradiated and annealed $0.6-\mu \mathrm{m} n$-channel devices and controls, as a function of drain bias, $V_{D}$, during a $10^{6}$-s hotcarrier stress. 
shown in Figure 4, where $\Delta V_{T H}$ is shown for an irradiated and annealed 0.6- $\mu \mathrm{m}$ device subjected to channel hot-carrier stress, along with the results of a standard subthreshold charge separation analysis, $\Delta V_{O T}$ and $\Delta V_{I T}$. The $\Delta V_{O T}$ component represents the "fixed" oxide trapped charge, and the $\Delta V_{I T}$ component represents the contribution made by the interface traps. In this case, the threshold voltage shift is clearly due almost entirely to activated interface traps. This result is typical of our results and it agrees with the data in the literature [10]. It should be noted that the maximum substrate bias condition $\left(V_{D}>V_{G}\right)$ for $n$-channel devices favors hole injection over electron injection. However, our overall goal was to compare our samples under conditions which would show an appreciable effect. Either increasing $V_{G}$ or decreasing $V_{D}$ in order to promote electron injection would decrease the maximum electric field in the channel, $E_{m}$, leading to significantly fewer hot carriers.

We also note that these results were obtained with $V_{D}=5 \mathrm{~V}$, whereas these parts are designed to run with $V_{D}=3.3 \mathrm{~V}$. The maximum localized field is proportional to the drain bias, so a larger $V_{D}$ will naturally lead to greater hot-carrier damage. (Our main motivation for increasing the drain bias was to obtain a large enough damage effect to study.) An illustration of this fact is seen in Figure 5, which shows the $\Delta V_{T H}$ responses of both irradiated/annealed and control devices, all with a channel length of $0.6 \mu \mathrm{m}$, as a function of drain bias, $V_{D}$. The pair with the large shift are the devices stressed with $V_{D}=5 \mathrm{~V}$. The devices stressed with the real-life bias of $3.3 \mathrm{~V}$ are shown hugging the abscissa. Just above those curves is the pair biased with $V_{D}=4$ $V$. Conventional values of $V_{D}$ produce negligible shifts; only overstressing produces significant aging. The reason is that the substrate current, $I_{S U B}$, increases rapidly as the drain bias increases to 5 or $6 \mathrm{~V}$. If an accurate measure of this substrate current can be made at higher drain biases, then the lifetime expectancy for a particular device stressed at normal values can be estimated.

In addition, these shifts are not as bad as they might seem, since these stresses were conducted with DC biases. Under normal operating conditions, these worst-case hot-carrier stressing conditions $\left(V_{D}>V_{G}>V_{T H}\right)$ do not occur with static biases in a real circuit, only when the device is being switched. Devices normally are switched only rarely, meaning that injection proceeds for only a small part of the lifetime of a device. Here the effects are quite negligible. We have observed no effect with $V_{D}=5 \mathrm{~V}$ when pulsing devices at $100 \mathrm{kHz}$. Evidence that at higher frequencies the delay in switching response causes the device to be biased so that hot-carrier effects are worse than under DC conditions has been the subject of some study [11]. All these considerations, however, miss the main point, which is that irradiated and annealed devices simulating $x$-ray lithography do not differ significantly from control devices representing conventional optical lithography processing.

\section{P-Channel Devices}

Initially, $n$-channel devices were the focus of hot-carrier effects studies since at longer channel lengths, only the higher impact ionization rate of electrons presented any problems [12].
As channel lengths have inexorably shrunk, $p$-channel devices have developed hot-carrier problems as well $[13,14]$.

Figure 6 compares the threshold voltage shifts of both control and irradiated/annealed 1.0- $\mu \mathrm{m}$-channel-length $p$-channel devices subjected to a channel hot-carrier stress for over $10^{6} \mathrm{~s}$. The scale is the same as that for the $n$-channel devices of Figures 1 to 3 . At this relatively long channel length, there is no real shift to speak of, nor is there any difference between the two devices. The devices were stressed in the same way as the $n$-channel devices, with negative values replacing positive bias values for both gate and drain. The $p$-channel device response generally depends on the magnitude of the gate current $[15,16]$, $I_{G}$, which is much smaller in magnitude than $I_{S U B}$, and thus hard to measure. But the peak $I_{G}$ value is to be found at about this gate bias for $p$-channel devices [17].

Figure 7 shows the results of a similar channel hot-carrier stress for devices with $0.6-\mu \mathrm{m}$ channel lengths. In this case, there was some discrepancy between the results, so two pairs of irradiated/annealed and control devices are shown. In one case the control exhibits a smaller $\Delta V_{T H}$ and in the other a slightly larger shift. We conclude that there is no significant difference between the device responses.

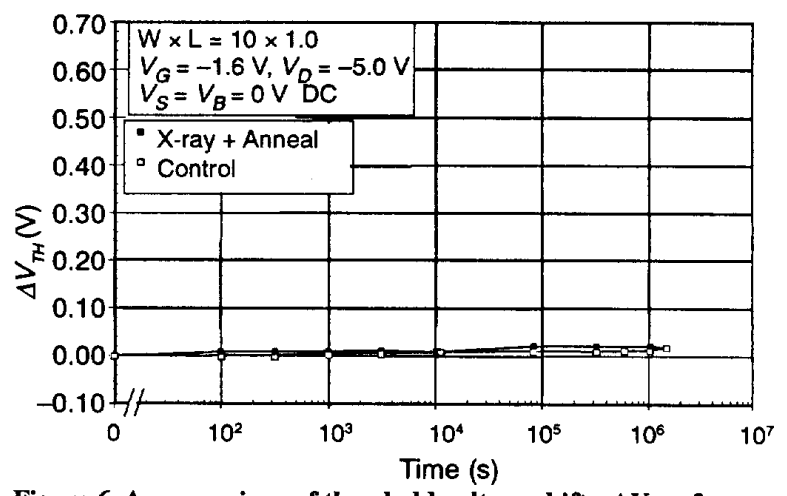

Figure 6. A comparison of threshold voltage shifts, $\Delta V_{T H}$, for both irradiated and annealed $1.0-\mu \mathrm{m} p$-channel devices and controls, during a $10^{6}$-s hot-carrier stress.

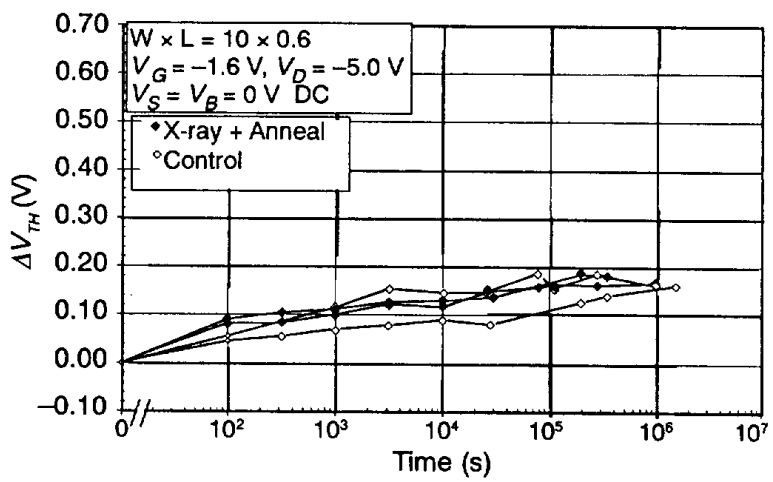

Figure 7. A comparison of threshold voltage shifts, $\Delta V_{T H}$, for both irradiated and annealed $0.6-\mu \mathrm{m} p$-channel devices and controls, during a $10^{6}$-s hot-carrier stress. 
Figure 8 displays the $\Delta V_{T H}$ response for the devices in Figures 6 and 7 , along with intermediate channel-length devices stressed in a similar manner. Once again, the shortest and longest channel length devices serve as the limits for all the devices, displaying the largest and smallest shifts, respectively. The $0.7-$ and 0.8- $\mu \mathrm{m}$ channel-length devices oddly show a larger shift for the control devices. We would in this case conclude that the $x$ ray lithography simulation certainly does no worse than optical lithography.

In all cases, a relatively large initial shift is followed by a general saturation of $\Delta V_{T H}$, which is consistent with results in the literature [18]. The channel hot holes are believed to generate electron-hole pairs via impact ionization, and these electrons are then trapped in the gate oxide near the drain. The trapped negative charge eventually serves to inhibit further trapping. That electron trapping is the problem can be seen in Figure 9, where the threshold voltage shift is once more separated into oxide trap and interface trap components-in this case for an irradiated and annealed $0.6-\mu \mathrm{m}$ channel-length device. The positive $\Delta V_{T H}$ is due mostly to a positive $\Delta V_{O T}$ indicating trapped negative charge: electrons.

Finally, we have presented only $\Delta V_{T H}$ results so far, and based all of our conclusions upon them. We also measured the maximum transconductance, $g_{m}$, for these devices. The $n$ -

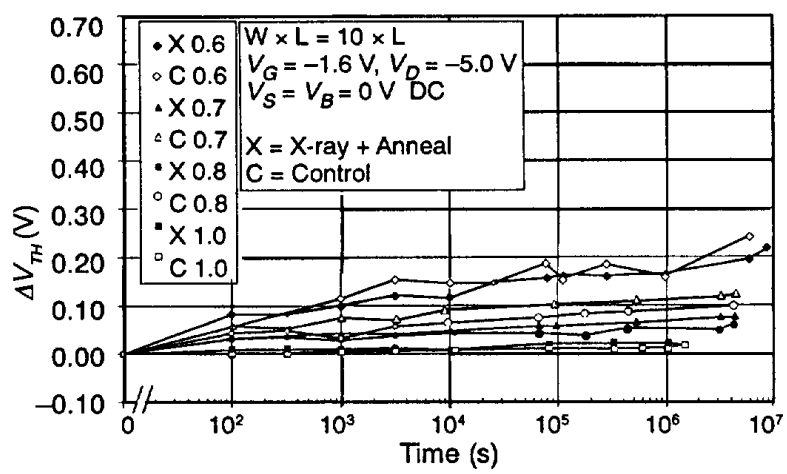

Figure 8. A comparison of threshold voltage shifts, $\Delta V_{T H}$, for both irradiated and annealed $p$-channel devices and controls, as a function of channel length, during a $10^{6}$-s hot-carrier stress.

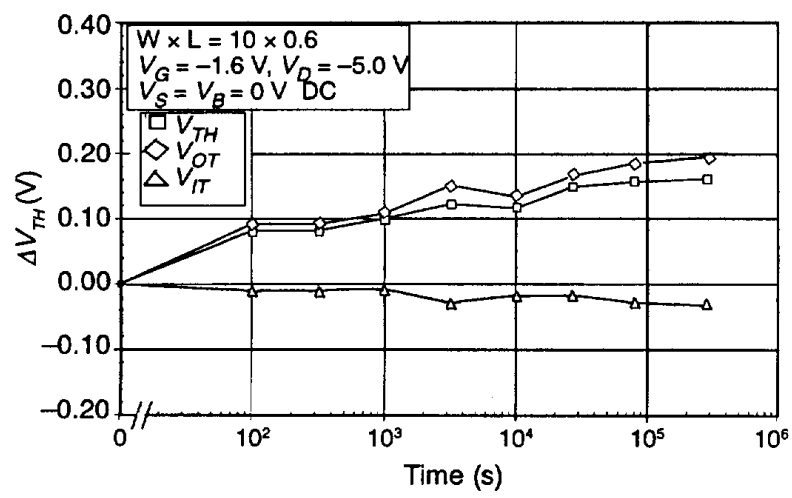

Figure 9. Subthreshold charge separation results for an irradiated and annealed $0.6-\mu \mathrm{m} p$-channel device subject to a channel hot-carrier stress for $10^{6} \mathrm{~s}$. channel devices exhibit a decrease in $g_{m}$, whereas it increases in $p$-channel devices over time. Figure 10 shows the results for both $n$ - and $p$-channel devices, as a function of channel length. Both irradiated/annealed and control devices are shown. These results are very similar to the $\Delta V_{T H}$ results of Figures 3 and 8 (the key for the responses of both the irradiated/annealed and control devices at various channel lengths is the same as those used in Figures 3 and 8 ). For the $n$-channel devices, the shorter channel-length devices exhibit a larger decrease in $g_{m}$, due to the larger electric field, $E_{m}$, present. However, in each case the control devices' responses nearly match those of the irradiated/ annealed devices. The results for the $p$-channel devices are clustered together, $g_{m}$ increasing between 5 and 15 percent. Once again, the control devices' responses match those of the irradiated/annealed devices.

\section{Conclusion}

Since the post-irradiation high-temperature anneal removes most of the radiation damage from the $\mathrm{x}$-ray exposure due to interface traps, $N_{I T}$, and to oxide trapped holes, $N_{O T}$, we might expect NETs (neutral electron traps) to be the only difference between our two sample sets (which reportedly do not anneal at $400^{\circ} \mathrm{C}[5]$ ). Our results suggest that NETs are not a problem.

Channel hot-carrier stressing does noticeably affect the threshold voltage (among other parameters, including decreases in drain current) in $n$-channel devices. However, these results are due to a growth in active interface traps and not trapped negative charge (filling of NETs). (Bias conditions which would promote electron trapping do not generate enough hot carriers to be a problem either.) More importantly, no significant difference was observed between the control devices and those exposed to simulated $\mathrm{x}$-ray lithography processing. As expected, the increase in $\Delta V_{T H}$ correlated with a decrease in the drawn channel length. But even at the shortest channel lengths, the shifts are quite small under more realistic bias conditions (smaller drain biases under DC and AC stressing).

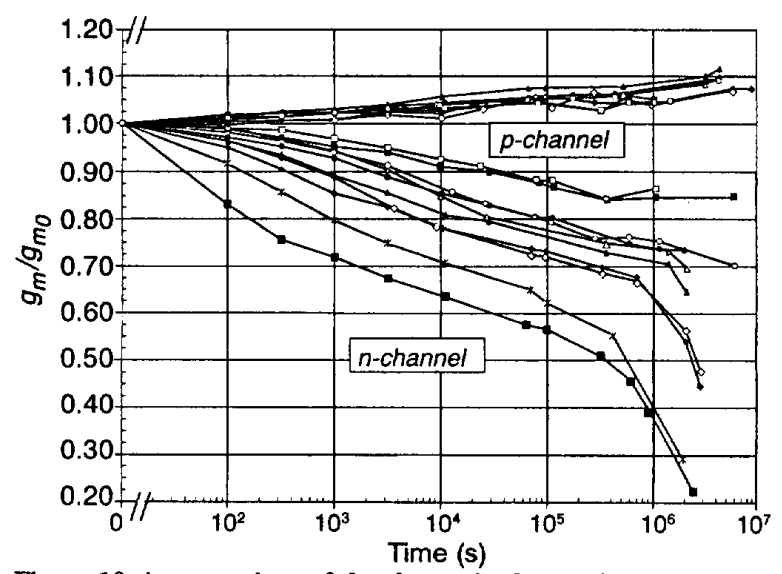

Figure 10. A comparison of the change in the maximum transconductance, $g_{m}$, for both irradiated/annealed $n$-channel (decreasing $g_{m}$ ) and $p$-channel (increasing $g_{m}$ ) devices and controls, as a function of channel length, during a $10^{6}$-s hotcarrier stress. 
The $p$-channel devices with submicron channel lengths had small positive threshold voltage shifts due in this case to trapped negative charge. However, these results are probably not due to process ( $x$-ray) induced NETs, since no differences were observed between control and irradiated devices. Again, these results are for worst-case DC bias stressing.

In summation, neither $n$ - or $p$-channel devices showed any significant differences between the control devices and those exposed to simulated $\mathrm{x}$-ray lithography processing.

\section{REFERENCES}

[1] F. B. McLean, H. E. Boesch, and T. R. Oldham, Electron-Hole Generation, Transport, and Trapping in $\mathrm{SiO}_{2}$, Chapter 3 of Ionizing Radiation Effects in MOS Devices and Circuits, T. P. Ma and P. V. Dressendorfer, eds, Wiley Interscience, New York (1989).

[2] P. S. Winokur, Radiation-Induced Interface Traps, Chapter 4 of Ionizing Radiation Effects in MOS Devices and Circuits, T. P. Ma and P. V.Dressendorfer, eds, Wiley Interscience, New York (1989).

[3] T. R. Oldham, F. B. McLean, H. E. Boesch, and J. M. McGarrity, An Overview of Radiation-Induced Interface Traps in MOS Structures, Semiconductor Science and Technology, 4, 986 (1989).

[4] M. Walters and A. Reisman, Radiation-Induced Neutral Electron Trap Generation in Electrically Biased Insulated Gate Field Effect Transistor Gate Insulators, J. Electrochem. Soc., 138, 27562762 (1991).

[5] A. J. Lelis and T. R. Oldham, X-Ray Lithography Effects on MOS Oxides, IEEE Trans. Nucl. Sci., NS-39, 2204-2210 (1992).

[6] P.S. Winokur, J. R. Schwank, P. J. McWhorter, P. V. Dressendorfer, and D. C. Turpin, Correlating the Radiation Response of MOS Capacitors and Transistors, IEEE Trans. Nucl. Sci., NS-31, 14531460 (1984).

[7] J. Y.-C. Sun, J. R. Maldonado, M. D. Rodriguez, J. Laskar, and D. S. Zicherman, Effects of X-Ray Irradiation of the Channel HotCarrier Reliability of Thin-Oxide n-Channel MOSFETs, Ext. Abst. 18th (Intl) Conf. Solid State Dev. Mater. (Tokyo), 479-482 (1986).
[8] E. Takeda, H. Kume, T. Toyabe, and S. Asai, Submicrometer MOSFET Structure for Minimizing Hot-Carrier Generation, IEEE Trans. Elect. Dev., ED-29, 611-618 (1982).

[9] T. Y. Chan, P. K. Ko, and C. Hu, Dependence of Channel Electric Field on Device Scaling, IEEE Elect. Dev. Lett., EDL-10, 551553 (1985).

[10] F.-C. Hsu and S. Tam, Relationship Between MOSFET Degradation and Hot-Electron-Induced Interface-State Generation, IEEE Elect. Dev. Lett., EDL-5, 50-52 (1984).

[11] K. R. Mistry and B. Doyle, $A C$ versus DC Hot-Carrier Degradation in n-Channel MOSFET's, IEEE Trans. Elect. Dev., ED-40, 96-104 (1993).

[12] J. Y. Chen, CMOS-The Emerging VLSI Technology, IEEE Circuits and Devices Magazine, 16-31 (March 1986).

[13] C. C.-H. Hsu, L. K. Wang, M. R. Wordeman, and T. H. Ning, HotElectron Induced Instability in 0.5- on p-Channel MOSFETs Patterned Using Synchrotron X-Ray Lithography, IEEE Elect. Dev. Lett., EDL-10, 327-329 (1989).

[14] C. C.-H. Hsu, L. K. Wang, J. Y.-C. Sun, M. R. Wordeman, and T. H. Ning, Radiation Damage and its Effect on Hot-Electron Induced Instability of 0.5- $\mu \mathrm{m}$ CMOS Devices Patterned Using Synchrotron X-Ray Lithography, J. Electron. Mater., 19, 721-725 (1990).

[15] T.-C. Ong, K. Seki, P. K. Ko, and C. Hu, P-MOSFET Gate Current and Device Degradation, IEEE Reliability Physics Symposium, 178-182 (1989).

[16] D.-H. Huang, E. E. King, and L. J. Palkuti, Improved Method for Evaluating Hot-Carrier Aging in p-Channel MOSFET's, IEEE Reliability Physics Symposium, 38-42 (1993).

[17] K. K. Ng and G. W. Taylor, Effects of Hot-Carrier Trapping in nand p-Channel MOSFET's, IEEE Trans. Elect. Dev., ED-30,871876 (1983).

[18] M. Koyanagi, A. G. Lewis, R. A. Martin, T.-Y. Huang, and J. Y. Chen, Hot-Electron-Induced Punchthrough (HEIP) Effect in Submicrometer PMOSFET's, IEEE Trans. Elect. Dev., ED-34, 839-844 (1987). 